\title{
Microbial Opsins: A Family of Single-Component Tools for Optical Control of Neural Activity
}

\author{
Ofer Yizhar, Lief Fenno, Feng Zhang, Peter Hegemann, and Karl Diesseroth
}

\section{INTRODUCTION}

Members of the microbial opsin gene family have emerged recently as crucial tools for "optogenetics," a new neuroscience technology. "Optogenetics" can be defined as the integration of optics and genetics to control well-defined events (such as action potentials) within specified cells (such as a targeted class of projection neurons) in living tissues (such as the brains of freely behaving mammals). In this article, we focus on the diversity of the microbial opsin genes and the structure-function properties of their corresponding proteins.

\section{BACKGROUND}

Light is the ultimate source of energy and a major source of environmental information, for almost all living things. For this reason, the efficient capture and use of photons is a widespread and exquisitely evolved process that is represented across all kingdoms of life. Microorganisms have evolved particularly diverse light-dependent systems to generate energy, avoid maladaptive situations, and identify environments suitable for nutrition and symbiosis, in many cases using different members of the seven-transmembrane-segment opsin gene family. For example, using microbial opsin genes (which include bacteriorhodopsins, halorhodopsins, sensory rhodopsins, and channelrhodopsins) (Fig. 1), prokaryotes can transduce light to shift proton gradients, modulate chloride balance, or switch flagellar motor direction, whereas motile algae transduce light to change flagellar beating to direct locomotion toward environments optimally illuminated for their photosynthetic requirements.

The central operating principle of these elegant molecular machines for ion flux is their unitary, simple nature: They combine the two tasks of light sensation and ion flux into a single protein (with a bound small organic cofactor) encoded by a single gene (Oesterhelt and Stoeckenius 1971). Because of this unitary operating principle, the diverse and efficient microbial opsins have (in retrospect) always had potential for application to neuroscience. However, this potential remained latent for several decades after discovery; many technical challenges needed to be overcome for this possibility to be realized. It has since been shown that expressing microbial opsin genes in neurons can enable millisecond-precision optical control of genetically targeted neuronal populations in mammals (Boyden et al. 2005; Li et al. 2005; Ishizuka et al. 2006; Adamantidis et al. 2007; Zhang et al. 2007, 2008).

This approach, which we termed "optogenetics" (Deisseroth et al. 2006), is fundamentally distinct from earlier designs for optical control (Kramer et al. 2005; Miesenböck and Kevrekidis 2005). It capitalizes on the portability of microbial opsins in efficiently coding for light sensation and effector function within a single gene, and the endogenous presence of the essential organic cofactor retinal in all vertebrate tissues tested, including brain. Thus, the single-component nature of the system is preserved, and no exogenous chemicals need to be added. Furthermore, neurons transduced with opsins are responsive to visible-spectrum light with biologically well-tolerated wavelengths and intensities. Also, the ability to deliver temporally precise light pulses to deep mammalian tissues in vivo with novel, engineered optical devices provides a great degree of experimental versatility. Together, these properties

Adapted from Imaging in Neuroscience (ed. F Helmchen, 


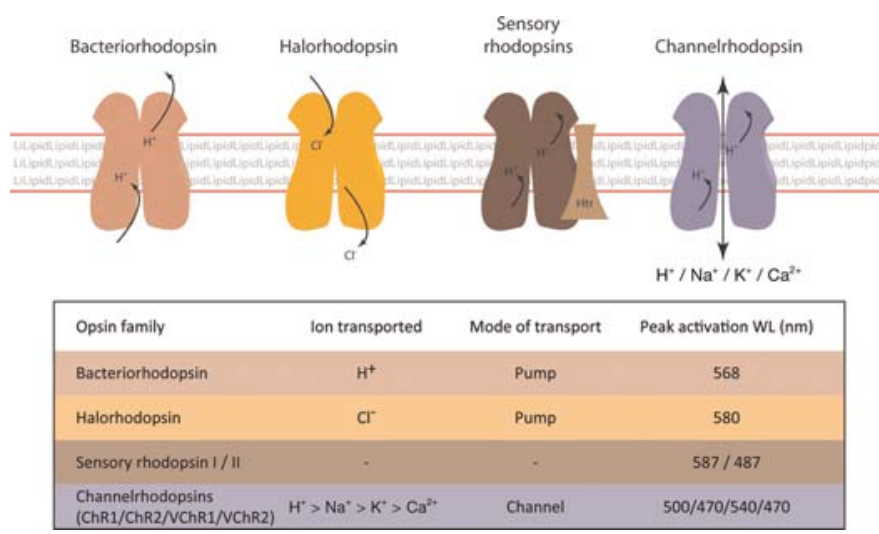

FIGURE 1. Overview of type I microbial rhodopsins. Bacteriorhodopsin and proteorhodopsins pump protons from the cytoplasm to the extracellular medium, whereas halorhodopsins pump chloride into the cytoplasm; all three change the membrane voltage in the same direction. In sensory rhodopsins, the internal proton movement is converted to conformational changes that drive signaling cascades. Algal channelrhodopsins flux cations across the membrane in both directions but always along the electrochemical gradient of the transported ions. In sensory and channelrhodopsins, proton translocation within the protein is required for efficient photocycle progression, but these protons are not necessarily exchanged between the intra- and extracellular space.

have triggered a surge of interest not only into immediate neuroscience applications, but also into using genomics and molecular engineering to expand the repertoire of tools and generate novel classes of functionality for neuroscience. Here we focus on the fundamentals of microbial opsin gene relationships and the structure-function properties of the ancient and powerful class of proteins encoded by these genes.

\section{COMMON PRINCIPLES OF OPERATION}

All opsins require retinal, a vitamin A-related organic cofactor that serves as the antenna for photons, to function (Fig. 2A); when retinal is bound, the functional opsin proteins are termed rhodopsins. After retinal has diffused into the binding pocket of the seven-transmembrane-helix oval, it is covalently attached to a conserved lysine residue of helix 7 by formation of a protonated retinal Schiff base $\left(\mathrm{RSBH}^{+}\right.$) (Fig. 2A). The ionic environment of the $\mathrm{RSBH}^{+}$, defined by the residues of the binding pocket, dictates the spectral and kinetic characteristics of each individual opsin. Upon absorption of a photon, retinal isomerizes and triggers a wave of conformational changes within the opsin partner. The photoisomerized retinal is the trigger for all subsequent structural rearrangements and all activities these proteins perform.

Opsin genes are divided into two distinct superfamilies: microbial-type opsins (type I) and animal-type opsins (type II). Although both families encode seven-transmembrane structures, sequence homology between the two is practically nonexistent; homology within families, however, is high (25\%-80\% residue similarity) (Man et al. 2003). Type I opsin genes are found in prokaryotes, algae, and fungi, where they control diverse functions such as phototaxis, energy storage, development, and retinal biosynthesis (Spudich 2006). Type II opsin genes are present only in higher eukaryotes and are mainly responsible for vision, but also play roles in circadian rhythm and pigment regulation (Sakmar 2002; Shichida and Yamashita 2003).

Type II opsins encode G-protein-coupled receptors (GPCRs). In the dark, these proteins bind retinal in the 11-cis configuration (Fig. 2A, bottom). Upon illumination, retinal isomerizes to the all-trans configuration (Fig. 2A, top) and initiates the reactions that underlie the visual phototransduction second messenger cascade. After photoisomerization, the retinal-protein linkage is hydrolyzed; free all-trans retinal then diffuses out of the protein and is replaced by a fresh 11-cis retinal molecule for another round of signaling (Hofmann et al. 2009). In contrast, type I opsins encode proteins that use retinal in the all-trans configuration, which isomerizes upon photon absorption to the 13-cis configuration (Fig. 2A, middle). Unlike the situation with type II rhodopsins, the activated retinal molecule in type I rhodopsins does not dissociate from its opsin protein, but thermally reverts to the all-trans state while 
A

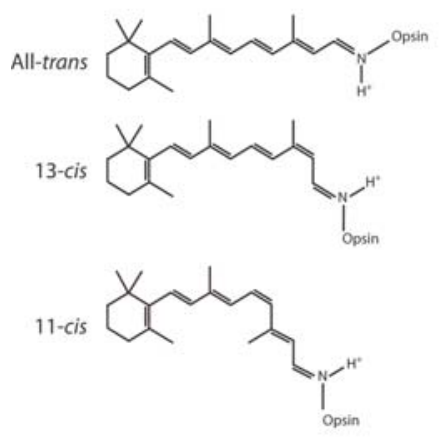

B

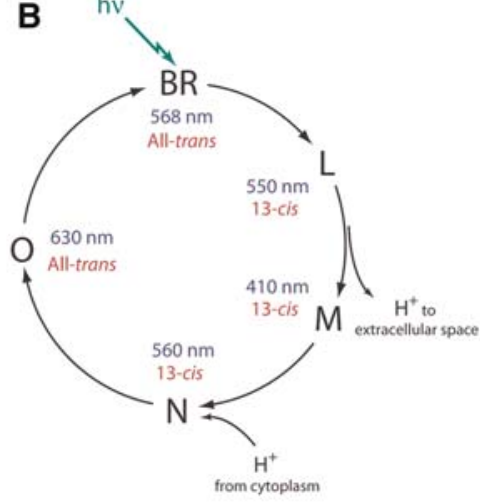

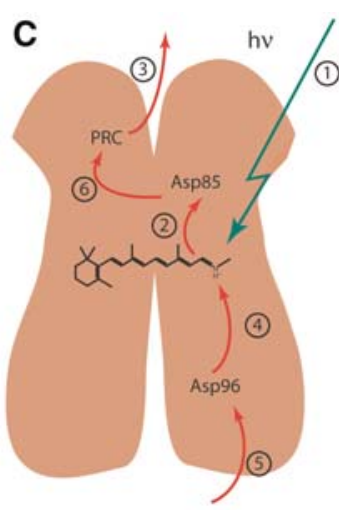

D BR and ChR

HR
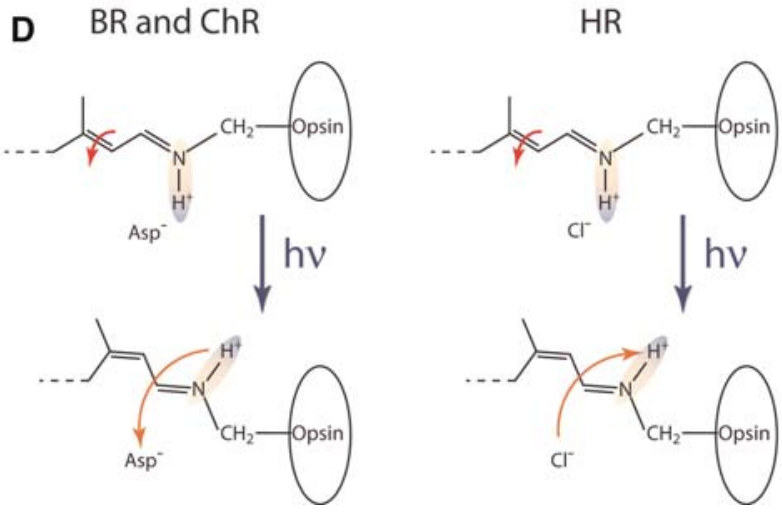

FIGURE 2. Photoreaction mechanisms. (A) Distinct configurations of the retinal Schiff base (RSB). (Top) Retinal in the alltrans state, as found in the dark-adapted state of microbial rhodopsins and in the light-activated forms of type II rhodopsins of higher eukaryotes. (Middle) Upon absorbing a photon, protonated microbial RSB converts to the 13-cis retinal configuration. (Bottom) 11-Cis retinal is found only in type II rhodopsins, where it binds to the opsin in the dark state before isomerizing to the all-trans position after photon absorption. (B) The photocycle of bacteriorhodopsin (BR) is initiated from the dark-adapted state; photon absorption activates a sequence of photochemical reactions and structural changes represented by the indicated photointermediates. Also shown are the configuration of the RSB at each step (red) and the wavelength at which each intermediate absorbs light maximally (blue). (C) Summary of proton transport reactions during the BR photocycle. Photon absorption (1) initiates the conformational switch in the RSB; leading to (2) transfer of a proton to Asp 85; (3) release of a proton from the proton release complex (PRC); (4) reprotonation of the RSB by Asp 96; (5) uptake of a proton from the cytoplasm to reprotonate Asp 96; and (6) the reprotonation of the PRC from Asp 85. (D) Light-induced switching of dipole orientation in response to photon absorption in $\mathrm{BR}, \mathrm{ChR}$, and HR. The configuration switch triggers the transfer of the RSB proton to Asp 85 or Glu 123 in BR or ChR2, respectively. In HR, dipole switching facilitates the transfer of a $\mathrm{Cl}^{-}$ion from the cavity formed between the RSB and Thr 143, to a $\mathrm{Cl}^{-}$binding site cytoplasmic to the RSB, enabling the key transport steps of these pumps. (For color figure, see doi: 10.1101/pdb.top102 online at www.cshprotocols.org.)

maintaining a covalent bond to its protein partner (Haupts et al. 1997). Type I opsins encode distinct categories of protein, discussed in more detail below.

\section{LIGHT-GATED ION PUMPS: BACTERIORHODOPSINS, PROTEORHODOPSINS, HALORHODOPSINS}

Bacteriorhodopsin (BR) was described by Oesterhelt and Stoeckenius (1971) as the first singlecomponent transmembrane protein known to be capable of actively translocating ions across the cell membrane in the light; it was also recently shown to function as an optogenetic tool (Gradinaru et al. 2010). BR is highly expressed in haloarchaeal membranes under low oxygen conditions to maintain a proton gradient across the membrane when respiration ceases (Oesterhelt and Stoeckenius 1971) and to drive ATP synthesis and maintain cellular energetics (Racker and Stoeckenius 1974; Michel and Oesterhelt 1976). Early work on BR noted that the protein cycles between states that absorb light at $568 \mathrm{~nm}$ and $400 \mathrm{~nm}$ (Oesterhelt and Hess 1973). Further research into the mechanism of this 
reaction led to detailed descriptions of a more complete photocycle, in which BR shuttles among several functional states called photointermediates (Fig. 2B; Stoeckenius et al. 1977). Each photointermediate has a distinct spectral signature and represents a separate reaction in the proton translocation process. The photocycle is essentially comprised of a series of proton transfer reactions, each with a unique role in the process of routing protons from the intracellular to the extracellular space (Luecke et al. 1999).

Photon absorption by BR initiates the isomerization of bound retinal from the all-trans to the 13-cis configuration (Fig. 2B), thereby triggering a series of proton-transfer reactions that constitute the proton translocation mechanism of BR (Fig. 2C). The conformational shift and changing dipole of the $\mathrm{RSBH}^{+}$ raises its $\mathrm{p} K$, resulting in release of the RSB proton and generating the $\mathrm{M}$ intermediate, named after the active state of vertebrate rhodopsin, which maximally absorbs light at $410 \mathrm{~nm}$; the released proton is accepted by the nearby Asp 85, which is part of the counterion complex. A distinct proton is then released from a group of amino acids near the extracellular side of the opsin, termed the proton release complex (PRC), out to the extracellular medium. At this point, the RSB captures a proton from Asp 96, and the protein enters the $N$ intermediate, which absorbs at $560 \mathrm{~nm}$. Asp 96 is then reprotonated by resident cytoplasmic protons, which is the key step in modulating cellular proton concentration. During this Asp 96 proton uptake, the Schiff base reisomerizes back to all-trans (defining the $\mathrm{O}$ state), which absorbs at $630 \mathrm{~nm}$. In the final step, Asp 85 transfers its proton to the PRC, thereby completing one full photocycle and returning BR to its original state. The net outcome is that, upon absorption of a single photon, BR releases one proton to the extracellular milieu and absorbs a second proton from the cytoplasm in a sequence of steps that are spatially disconnected. As discussed below, amino acids involved in the proton translocation reactions are commonly conserved across other type I opsins with homology to BR and appear to function as a conserved pathway for proton translocation.

Light-driven proton pumps are not restricted to archaea but are also found in eubacteria. Marine proteobacteria represent a diverse and widespread class of eubacteria found from the Arctic to the Antarctic Oceans, including equatorial and temperate waters at many depths (Sabehi et al. 2007; Atamna-Ismaeel et al. 2008). Their associated opsin genes encode proteorhodopsins (PRs), with proton pump photocycles similar to that of BR (Váró et al. 2003). However, although PRs can be used to drive ATP production, some proteobacteria do not grow faster in response to increased light, and others have photocycles too slow to contribute significantly to cellular energy production, suggesting other functions for PR (Spudich 2006). The spectra of marine PRs are tuned to ocean depth and latitude, and PRs share a high degree of sequence similarity across species, suggesting that genomic techniques might contribute to our understanding of opsin spectral tuning (Man et al. 2003). Interestingly, absorption variances between blue and green wavelengths can depend on a single amino acid residue (Béjà et al. 2001; Man et al. 2003), but attempts to transfer mutations conferring spectral tuning from PR to other microbial opsins have met with limited success (Yoshitsugu et al. 2009). Nevertheless, the utility of single-amino-acid modifications in shifting absorption spectra has been exploited in vitro to screen randomly mutagenized PR libraries, yielding both red- and blue-shifted mutants (Kim et al. 2008).

Although BR and PR can certainly generate outward (i.e., hyperpolarizing) currents that could in principle be useful for neuronal inhibition, these pumps can produce local juxtamembranous (especially extracellular) pH changes (Chow et al. 2010; Gradinaru et al. 2010). In contrast, halorhodopsin (HR) is a distinct class of outward-current-generating archaeal opsin that uses chloride ions (Matsuno-Yagi and Mukohata 1977). HR is an electrogenic ion pump that controls gradients across the cell membrane by transporting chloride ions from the extracellular medium into the cell (Schobert and Lanyi 1982; Bamberg et al. 1984). Its primary photocycle, although qualitatively similar to that of BR, does not show $\mathrm{RSBH}^{+}$deprotonation (Oesterhelt et al. 1985; Essen 2002) because of a single amino acid change at the acceptor Asp (Fig. 3A): After the light-induced retinal isomerization and $\mathrm{RSBH}^{+}$dipole switch, the proton cannot be released because the acceptor Asp has been replaced by Thr. Instead, a $\mathrm{Cl}^{-}$ion is shuffled from the external side of the $\mathrm{RSBH}^{+}$chromophore to the internal side, which is then funneled in subsequent steps to bulk intracellular phase; the external $\mathrm{Cl}^{-}$binding site is refilled by an extracellular $\mathrm{Cl}^{-}$ion (Fig. 2D; Kolbe et al. 2000).

Halorhodopsin was the first archaeal protein to be successfully applied in neuroscience, when an experimental screen (Zhang et al. 2007) revealed that the best-known HR (from Halobacterium salinarum) failed to maintain stable photocurrents when expressed heterologously, but the HR from the less halophilic Natronomonas pharaonis (Scharf and Engelhard 1994) expressed and functioned in metazoan neurons even in vivo and could block cellular activity and action potentials by 
A

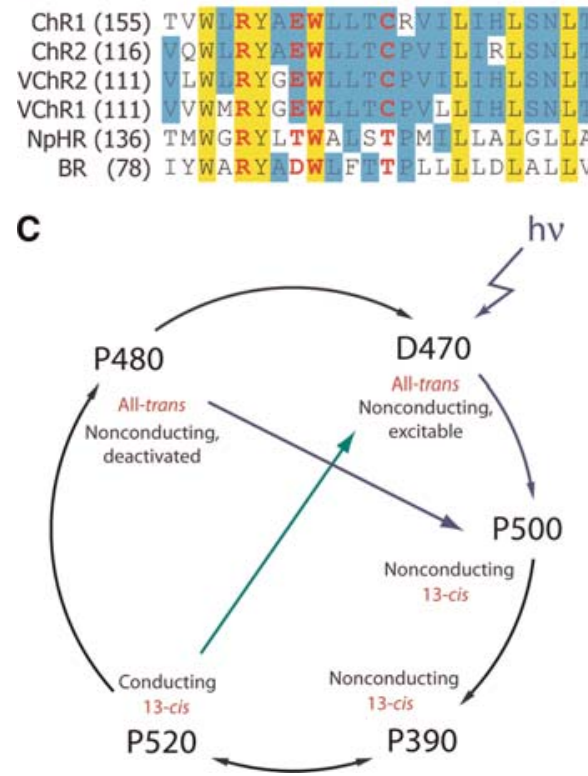

B

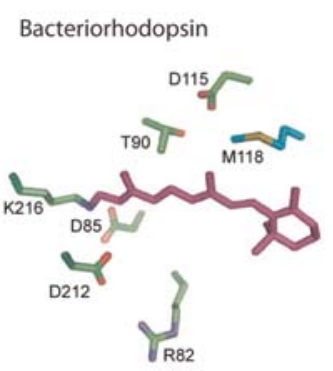

Channelrhodopsin-2

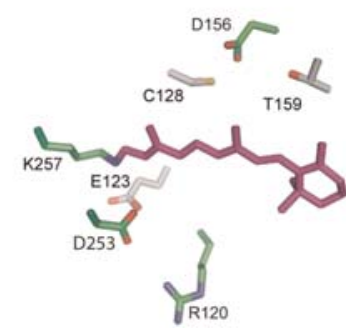

FIGURE 3. Structural and functional homologies of BR and ChR. (A) Sequence homology between the algal ChRs and BR within the third transmembrane helix. Identical and conserved residues are highlighted in yellow and blue, respectively; amino acids interacting with the chromophore are shown in red. In BR, R82 functions as a connector between counterion and the PRC; D85 is the counterion to which the RSB proton is transferred; W86 provides hydrophobicity for retinal binding; and the hydrogen bond between T90 on helix 3 and D115 on helix 4 (not shown) stabilizes the structure of the binding pocket and enables both residues to control rapid reprotonation and reisomerization of retinal to the all-trans state following a light pulse. (NpHR) Natronomonas pharaonis HR. (B) Anticipated homology in the retinal binding pocket between BR and ChR2. The BR pocket is shown based on structure 1KGB (Facciotti et al. 2001) with key amino acids that are involved in the proton transfer reaction. The ChR2 pocket was modeled after the BR structure, based on sequence homology in the relevant positions. (C) Model for the photocycle of ChR2. The RSB configuration and conductive state of each photocycle component are shown in red and black, respectively. The D470 dark state is converted by a light-induced isomerization of retinal via the early intermediate P500 and the transient intermediate P390 to the conducting state P520. The recovery of the D470 dark state proceeds either thermally via the nonconducting P480 intermediate or photochemically via possible short-lived intermediates (green arrow). The late or desensitized P480 state can also be activated (blue arrow) to yield the early intermediate P500. (For color figure, see doi: 10.1101/pdb.top102 online at www.cshprotocols.org.)

hyperpolarizing neurons with electrogenic inward $\mathrm{Cl}^{-}$currents. Inactivation is modest, allowing stable, step-like currents over many tens of minutes in response to steady yellow light (Zhang et al. 2007). Because of the stoichiometry of only one transported $\mathrm{Cl}^{-}$per photocycle, robust expression is required. This is best achieved by addition of mammalian membrane trafficking signals (Gradinaru et al. 2008), which could become a common theme when adapting prokaryotic membrane proteins for expression in mammalian cells. Under these conditions, nanoampere-scale currents and hyperpolarizations of $>100 \mathrm{mV}$ can be achieved, with responsivity to yellow or even red light pulses (Gradinaru et al. 2010).

Following the discovery of $\mathrm{BR}$ and $\mathrm{HR}$, two more retinal-binding proteins were identified from H. salinarum. Sensory rhodopsin I (SR-I) and phoborhodopsin (now termed SR-II) were identified initially as behaviorally relevant photosensors (Hildebrand and Dencher 1975; Takahashi et al. 1985). SRs use a photocycle similar to that of BR (Spudich and Bogomolni 1984; Spudich 1998), except that lightinitiated conformational changes and internal proton movement within the opsin are used to activate a closely associated transducer molecule, Htr (Büldt et al. 1998; Chen and Spudich 2002). The activated transducer initiates a phosphorylation cascade that controls the directionality of the flagellar motor and facilitates phototaxis to either the green-yellow range of wavelengths (SR-I, peak absorption $587 \mathrm{~nm}$ ) or away from the blue range (SR-II, peak absorption $487 \mathrm{~nm}$ ) (Spudich and Bogomolni 1984; Spudich 2006). Because prokaryotic kinase cascades are fundamentally different from eukaryotic second messengers, opportunities to translate SR function to other systems are more complicated than 
with the ion conductance-regulating microbial opsins such as $\mathrm{HR}$, although SR proton pumping is possible (Haupts et al. 1996).

\section{LIGHT-GATED ION CHANNELS: CHANNELRHODOPSINS}

Continuing the theme of single-component microbial transmembrane ion conductance regulators, channelrhodopsin-1 (ChR1) was identified as a light-gated ion channel in Chlamydomonas reinhardtii, a green unicellular alga from temperate freshwater environments. Although it was originally identified as proton-selective (Nagel et al. 2002), a broader cation conductance for ChR1, including $\mathrm{Na}^{+}$and $\mathrm{K}^{+}$, was later described (Lin et al. 2009; Tsunoda and Hegemann 2009). Channelrhodopsin-2 (ChR2) was later discovered in the same organism: ChR2 also conducts protons, but its $\mathrm{Na}^{+}$and $\mathrm{K}^{+}$conductances are more than twofold greater than those of ChR1 (Nagel et al. 2003; Tsunoda and Hegemann 2009). ChR2 was found to express robustly enough in mammalian neurons that precisely timed light-driven action potentials were achievable (Boyden et al. 2005).

Both ChR1 and ChR2 bear some homology to BR and other type I rhodopsins; this homology is strong in the retinal-binding pocket and proton-conducting network (Hegemann et al. 2001; Nagel et al. 2002, 2003; Sineshchekov et al. 2002; Suzuki et al. 2003), suggesting related mechanisms of ionic phototransduction (Fig. 3B). Indeed, the photocycle of ChR2 is similar to that of BR but with different spectral characteristics, likely resulting from variations in the ionic environment near the RSB, larger conformational changes within the protein (Fig. 3C; Ritter et al. 2008; Radu et al. 2009), and possibly higher water content within the proton network. In ChR2, a dark-adapted state absorbing at $470 \mathrm{~nm}$ (D470) converts rapidly upon illumination to the conducting state (P520) via the short-lived photointermediates P500 and P390. Illumination of the open channel at this step with green light terminates the photocurrent (Bamann et al. 2008; Berndt et al. 2009) by photochemically shifting the channel back to a closed state, which could be the dark-adapted state (D470) or the light-adapted state (P480) (K Stehfest and P Hegemann, unpubl.), effectively resetting the photocycle.

This photocycle shortcut pathway could be relevant only at very high light intensities with wildtype ChR2, but acquires a special utility with certain molecularly engineered slow mutants (e.g., step function opsin genes, or SFOs) (Berndt et al. 2009). Indeed, as with the light-activated ion pumps, molecular engineering and genomic strategies have begun to bear fruit for enhancing function of the activated ion channels for particular purposes. The long history of structure-function mutagenesis with regard to photocycle kinetics and absorption spectra in BR, along with the high degree of similarity between BR and ChRs in the chromophore region, has been capitalized on to modify the ChR photocycle and extend the array of tools available to control neuronal activity. For example, SFO mutations at Cys 128 in ChR2 (homologous to Thr 90 in BR) (Perálvarez-Marin et al. 2004) give rise to a several-hundred-fold extension of the open state (P520) lifetime and resulting long-acting (step function-like) ion fluxes in ChR2 in response to single pulses of light. This property leads to useful bi-stable behavior in the form of sustained subthreshold activation of neurons lasting up to minutes (Berndt et al. 2009; Stehfest et al. 2010). A histidine-to-arginine mutation in the residue homologous to the proton donor Asp 96 in BR (i.e., H134R in ChR2) increases photocurrents, but also slows the deactivation of ChR2 photocurrents, impairing temporal resolution (Nagel et al. 2005; Gradinaru et al. 2007). Chimeric proteins containing fragments of ChR1 and ChR2 reduce inactivation and alter the overall expression in mammalian cells toward higher or lower expression, depending on the individual helices shuffled (Lin et al. 2009; Wang et al. 2009). Finally, modification of the proton acceptor (homologous to Asp 85 in BR) in the Glu 123 position (i.e., ChETA mutations E123T and E123A) (Gunaydin et al. 2010) leads to much more rapid channel closing and enables ultrafast optical control of spiking at least up to $200 \mathrm{~Hz}$, with reduced inactivation and improved overall performance.

Genomic strategies have also been productive. For example, novel ChRs have been identified by genomic search strategies in the colonial alga Volvox carteri, expanding the family of known ChRs to four members. Volvox ChR2 absorbs similarly to ChR2 with a maximum near $470 \mathrm{~nm}$, but the Volvox ChR1 (VChR1) absorption is strikingly red-shifted to a maximum at $540 \mathrm{~nm}$ (Fig. 1) and allows lightdriven spike firing at $589 \mathrm{~nm}$, which has profound advantages for optogenetic applications (Zhang et al. 2008; Kianianmomeni et al. 2009). Generally, light at longer wavelengths is less deleterious for biological tissue. Moreover, tools with diverse spectral tuning offer the potential of addressing multiple distinct neuronal populations that are anatomically intermixed, with different colors of light. Identification of natural opsins in this way, along with rational engineering for novel properties, will likely continue to be productive. 


\section{QUANTITATIVE CONSIDERATIONS: OPSIN BIOPHYSICAL PROPERTIES}

Many factors control the efficacy of microbial opsins expressed in heterologous systems, not the least of which are cell biological properties ranging from effective transcription, translation, and folding, to proper membrane trafficking and targeting. However, biophysical properties will in many cases be similarly important limiting factors. The most basic of these is the efficiency of light absorption, expressed by the extinction coefficient $\left(\varepsilon_{\max }\right)$. For rhodopsins, $\varepsilon_{\max }$ is typically between 50,000 and $70,000 \mathrm{M}^{-1} \mathrm{~cm}^{-1}$, less than that of chlorophyll $\left(100,000 \mathrm{M}^{-1} \mathrm{~cm}^{-1}\right)$ but more than that of most other biological chromophores such as flavins $\left(12,000 \mathrm{M}^{-1} \mathrm{~cm}^{-1}\right)$. Another important parameter is quantum efficiency $(\Phi$, the fraction of absorbed photons that are efficacious in driving the relevant conformational change), which varies between 0.3 and 0.7 depending on the opsin species.

Also worthy of detailed consideration is the turnover time of the photocycle, a critical figure of merit both for native function and for neuroscience applications. For the most active transporters (HR and BR), the photocycle turnover time is $\sim 10-20 \mathrm{msec}$. Turnover is slower for the blue PR ( $80-100 \mathrm{msec}$ ) (Wang et al. 2003), limiting the development of blue PRs for neuroscience applications. However, even these values come from measurements at zero membrane voltage; the turnover for the fast pumps at a more physiologically negative voltage slows dramatically from 10-20 msec to $100-400 \mathrm{msec}$ (Geibel et al. 2001). Thus, the more negative membrane voltage becomes, the slower the photocycle becomes, and more light is needed to achieve a given increment of hyperpolarization. Interestingly, this also means that by hyperpolarizing the membrane, the pump slows down its own photocycle, an effect that will be enhanced by changes in the electrochemical gradient of the transported ion. In general, however, pump direction is not actually inverted by physiologically achievable membrane potentials or ion gradients. Although such an inversion has been reported for PR, the driving force against the normal pump direction arose from extreme negative voltage and a strong $\mathrm{pH}$ gradient; under these conditions, the pump can become leaky as protons are forced onto an internal acceptor (Lörinczi et al. 2009). Generally, pump inversion per se is not expected, especially in physiological settings.

For ChRs the kinetic issues are different. Ion transport (i.e., current) is coupled to occupancy of the conducting state. This, in turn, is determined by light intensity and wavelength, extinction coefficient, and quantum efficiency, as well as by a new factor, the lifetime of the resulting conducting state, dictated by the kinetics of the P520-P480 transition (Fig. 3C). For temporally precise depolarization with a single flash, wild-type ChR2 is adequate. In contrast, because of its extended conducting-state lifetime, the H134R mutation of ChR2 noted above delivers approximately twofold higher photocurrents, although temporal precision is somewhat reduced as a result of moderately slower deactivation (Nagel et al. 2005; Gradinaru et al. 2007; Lin et al. 2009). The SFO variants of ChR2, with the greatly slowed deactivation described above (e.g., C128X) (Berndt et al. 2009), achieve chronic depolarization with even brief light delivery. Moreover, in principle, these variants can achieve the same maximal current magnitudes as wild-type ChR but at much lower light levels (Schoenenberger et al. 2009). For a 10-msec lifetime open state (Ritter et al. 2008), ChR2 would have to be excited 100 times per second for a half-saturating stable current. Assuming an absorption cross-section of $2 \times 10^{-20} \mathrm{~m}^{2}$, this would require $5 \times 10^{21}$ photons $/ \mathrm{m}^{2}$-sec (i.e., $5 \times 10^{12}$ photon $/ \mathrm{mm}$-msec or $2 \mathrm{~mW} / \mathrm{mm}^{2}$ ) chronically. However, as a result of the extended-lifetime conducting states, C128T needs 100 times less light and the $\mathrm{C} 128 \mathrm{~S}$ mutant more than 1000 times less chronic light delivery for the same activity (Berndt et al. 2009).

Accelerating steps in the photocycle can also present certain advantages. For high-frequency neuronal firing, both fast-on and fast-off kinetics are important, requiring fast formation and decay of the P520 photocycle intermediate. Only the ChETA mutations (e.g., ChR2-E123T or E123A) can provide this unique functionality at present. Total current per flash is slightly smaller than for wild-type ChR2 because of the shorter open time, but as long as good expression is achieved, this can be compensated for by slightly more intense or longer flashes (e.g., 2 msec instead of $1 \mathrm{msec}$ ) (Gunaydin et al. 2010). Inactivation must also be considered, which is fast but weak in ChR1 and slow but strong in ChR2. Inactivation is more pronounced at high voltage (i.e., weakly negative or positive) and approaches $70 \%$ of peak current at $\mathrm{pH}$ 7.5. If inactivation of the current becomes problematic, ChR-E123T (Gunaydin et al. 2010), the ChIEF variant (Lin et al. 2009), or ChR2-E90Q (Ritter et al. 2008) might be suitable.

In addition to conducting-state lifetime, the unitary conductance of a channelrhodopsin will also control efficacy, and estimates for this important value range from $30 \mathrm{fS}$ to $\sim 1 \mathrm{pS}$ for ChR2 (Feldbauer et al. 2009; Lin et al. 2009). In the living alga Chlamydomonas, half-maximal current is carried by $1 \times 10^{6}$ ions; if there were 10,000 ChRs per eyespot with quantum efficiency of 0.67 (as with BR), this would 
correspond to 100 ions per ChR (Harz et al. 1992) and a unitary conductance of $0.3 \mathrm{pS}$. More recent estimates of opsin number ( 100,000 per eyespot) (Sineshchekov et al. 2002; Berthold et al. 2008) put the ions per ChR at 10, and conductance therefore at $30 \mathrm{fS}$. Recently, this value has been supported by application of noise analysis to purified ChR2 (40 fS) (Feldbauer et al. 2009), although nonstationary fluctuation analysis had resulted in somewhat higher values (>100 fS) (Lin et al. 2009). Despite the uncertainties inherent in all of these methods, the resulting estimates are still reasonably close and together provide useful boundary conditions.

The size of the unitary conductance is also relevant to the prospect of achieving two-photon (2P)mediated control. Conventional 2P methods pose challenges in this regard, as small excitation volumes with raster-scanning $2 \mathrm{P}$ laser systems will typically not recruit sufficient numbers of small-conductance, rapidly deactivating molecules to, for example, drive spiking. Two main approaches are being developed to address this issue, and 2P optogenetic control can now be achieved. First, control of light delivery can be adapted to individual imaged cells to provide spatiotemporally suitable illumination patterns that will recruit sufficient conductance within a single cell and within a temporal window set by current deactivation properties (Rickgauer and Tank 2009). Second, engineered opsins (e.g., SFOs and related mutants in which the deactivation kinetics are altered) can facilitate recruitment of threshold levels of conductance within a given temporal window. In this way, 2P-based recruitment of sparsely distributed cell populations within living tissue can become practical, pointing to the value of continuing to probe the structure-function relationships of the microbial opsins.

The action spectrum is also important, and developing absorbance at longer wavelengths than ChR2 $\left(\lambda_{\max }=470 \mathrm{~nm}\right)$ is particularly useful for depth penetration, safety, and combinatorial experiments. ChR1 is slightly red-shifted but does not express well and is pH-dependent. VChR1 (Zhang et al. 2008) absorbs at markedly red-shifted wavelengths $\left(\lambda_{\max }=540 \mathrm{~nm}\right)$, and spiking can be driven even at $589 \mathrm{~nm}$ in hippocampal neurons, but expression levels are significantly lower than that of ChR2 in most host cells. The slower decay of the open state (Zhang et al. 2008) partially compensates, but improvement of VChR1 expression will be very important, and a strongly expressing channelrhodopsin that absorbs beyond $550 \mathrm{~nm}$ will outperform and be preferable to ChR2 in the future. Indeed, as with VChR1, the channelrhodopsin variants have diverse useful properties but also corresponding limitations. For example, the SFO mutants have reduced dynamic range. Moreover, because of SFO hypersensitivity and the long lifetime of intermediates, in vitro experiments should be maintained at low light to avoid unwanted activation by ambient light. When imaging is required, illumination should be restricted to nonactivating wavelengths ( $>560 \mathrm{~nm}$ ). Complete deactivation of the SFO prior to experiments with a long pulse of $590-\mathrm{nm}$ light will ensure that any baseline activity is eliminated and will enable full activation of the SFO with 470-nm light at the desired time (Schoenenberger et al. 2009; Stehfest et al. 2010).

\section{SUMMARY}

In this article, we have focused on the diversity of the microbial opsin genes and the structure-function properties of the corresponding proteins. Although much has been learned in applying the use of microbial opsins to neuroscience, technological challenges lie ahead, including development of variants with further red-shifted absorption or altered ion selectivity, as well as more complex activation schemes with multiple optogenetic proteins. Biochemical tools are also now available for specific GPCR pathways (Airan et al. 2009), and increasingly complex avenues of control and spectral separation will aid in the design of more refined experiments that do justice to the combinatorial complexity of the mammalian brain. Further rapid expansion of the optogenetic toolkit now will be a natural consequence not only of ever-increasing genomic and metagenomic resources, but also of molecular engineering spurred by increased understanding of the structure-function relationships of these ancient and powerful molecular machines.

\section{REFERENCES}

Adamantidis AR, Zhang F, Aravanis AM, Deisseroth K, de Lecea L. 2007. Neural substrates of awakening probed with optogenetic control of hypocretin neurons. Nature 450: 420-424.

Airan RD, Thompson KR, Fenno LE, Bernstein H, Deisseroth K. 2009. Temporally precise in vivo control of intracellular signalling. Nature 458: 1025-1029.

Atamna-Ismaeel N, Sabehi G, Sharon I, Witzel K-P, Labrenz M, Jürgens K, Barkay T, Stomp M, Huisman J, Béjà O. 2008. Widespread distribution of proteorhodopsins in freshwater and brackish ecosystems. ISME J 2: 656-662.

Bamann C, Kirsch T, Nagel G, Bamberg E. 2008. Spectral characteristics of the photocycle of channelrhodopsin-2 and its implication for channel function. J Mol Biol 375: 686-694.

Bamberg E, Hegemann P, Oesterhelt D. 1984. Reconstitution of halorhodopsin in black lipid membranes. Prog Clin Biol Res 164: 73-79. 
Béjà O, Spudich EN, Spudich JL, Leclerc M, DeLong EF. 2001. Proteorhodopsin phototrophy in the ocean. Nature 411: 786-789.

Berndt A, Yizhar O, Gunaydin LA, Hegemann P, Deisseroth K. 2009. Bi-stable neural state switches. Nat Neurosci 12: 229-234.

Berthold P, Tsunoda SP, Ernst OP, Mages W, Gradmann D, Hegemann P. 2008. Channelrhodopsin-1 initiates phototaxis and photophobic responses in Chlamydomonas by immediate light-induced depolarization. Plant Cell 20: 1665-1677.

Boyden ES, Zhang F, Bamberg E, Nagel G, Deisseroth K. 2005. Millisecond-timescale, genetically targeted optical control of neural activity. Nat Neurosci 8: 1263-1268.

Büldt G, Heberle J, Dencher NA, Sass HJ. 1998. Structure, dynamics, and function of bacteriorhodopsin. J Protein Chem 17: 536-538.

Chen X, Spudich JL. 2002. Demonstration of 2:2 stoichiometry in the functional SRI-Htrl signaling complex in Halobacterium membranes by gene fusion analysis. Biochemistry 41: 3891-3896.

Chow BY, Han X, Dobry AS, Qian X, Chuong AS, Li M, Henninger MA, Belfort GM, Lin Y, Monahan PE, et al. 2010. High-performance genetically targetable optical neural silencing by light-driven proton pumps. Nature 463: 98-102.

Deisseroth K, Feng G, Majewska AK, Miesenböck G, Ting A, Schnitzer MJ. 2006. Next-generation optical technologies for illuminating genetically targeted brain circuits. J Neurosci 26: 10380-10386.

Essen L-O. 2002. Halorhodopsin: Light-driven ion pumping made simple? Curr Opin Struct Biol 12: 516-522.

Facciotti MT, Rouhani S, Burkard FT, Betancourt FM, Downing KH, Rose RB, McDermott G, Glaeser RM. 2001. Structure of an early intermediate in the $\mathrm{M}$-state phase of the bacteriorhodopsin photocycle. Biophys / 81: 3442-3455.

Feldbauer K, Zimmermann D, Pintschovius V, Spitz J, Bamann C, Bamberg E. 2009. Channelrhodopsin-2 is a leaky proton pump. Proc Natl Acad Sci 106: 12317-12322.

Geibel S, Friedrich T, Ormos P, Wood PG, Nagel G, Bamberg E. 2001. The voltage-dependent proton pumping in bacteriorhodopsin is characterized by optoelectric behavior. Biophys / 81: 2059-2068.

Gradinaru V, Thompson KR, Zhang F, Mogri M, Kay K, Schneider MB, Deisseroth K. 2007. Targeting and readout strategies for fast optical neural control in vitro and in vivo. I Neurosci 27: 14231-14238.

Gradinaru V, Thompson KR, Deisseroth K. 2008. eNpHR: A Natronomonas halorhodopsin enhanced for optogenetic applications. Brain Cell Biol 36: 129-139.

Gradinaru V, Zhang F, Ramakrishnan C, Mattis I, Prakash R, Diester I, Goshen I, Thompson KR, Deisseroth K. 2010. Molecular and cellular principles for diversifying and extending optogenetics. Cell 141: $1-12$.

Gunaydin L, Yizhar O, Berndt A, Sohal VS, Deisseroth K, Hegemann P. 2010. Ultrafast optogenetic excitation. Nat Neurosci 13: 387-392.

Harz H, Nonnengässer C, Hegemann P. 1992. The photoreceptor current of the green alga Chlamydomonas. Philos Trans $R$ Soc Lond B Biol Sci 338: 39-52.

Haupts U, Bamberg E, Oesterhelt D. 1996. Different modes of proton translocation by sensory rhodopsin I. EMBO / 15: 1834-1841.

Haupts U, Tittor J, Bamberg E, Oesterhelt D. 1997. General concept for ion translocation by halobacterial retinal proteins: The isomerization/switch/transfer (IST) model. Biochemistry 36: 2-7.

Hegemann P, Fuhrmann M, Kateriya S. 2001. Algal sensory photoreceptors. / Phycol 37: 668-676.

Hildebrand E, Dencher N. 1975. Two photosystems controlling behavioural responses of Halobacterium halobium. Nature 257: 46-48.

Hofmann KP, Scheerer P, Hildebrand PW, Choe H-W, Park JH, Heck M, Ernst OP. 2009. A G protein-coupled receptor at work: The rhodopsin model. Trends Biochem Sci 34: 540-552.

Ishizuka T, Kakuda M, Araki R, Yawo H. 2006. Kinetic evaluation of photosensitivity in genetically engineered neurons expressing green algae light-gated channels. Neurosci Res 54: 85-94.

Kianianmomeni A, Stehfest K, Nematollahi G, Hegemann P, Hallmann A. 2009. Channelrhodopsins of Volvox carteri are photochromic proteins that are specifically expressed in somatic cells under control of light, temperature, and the sex inducer. Plant Physiol 151: 347-366.

Kim SY, Waschuk SA, Brown LS, Jung KH. 2008. Screening and characterization of proteorhodopsin color-tuning mutations in Escherichia coli with endogenous retinal synthesis. Biochim Biophys Acta 1777: 504-513.

Kolbe M, Besir H, Essen L-O, Oesterhelt D. 2000. Structure of the lightdriven chloride pump halorhodopsin at $1.8 \AA$ resolution. Science 288: 1390-1396.

Kramer RH, Chambers JJ, Trauner D. 2005. Photochemical tools for remote control of ion channels in excitable cells. Nat Chem Biol 1: $360-365$.

Li X, Gutierrez DV, Hanson MG, Han J, Mark MD, Chiel H, Hegemann P, Landmesser LT, Herlitze S. 2005. Fast noninvasive activation and inhibition of neural and network activity by vertebrate rhodopsin and green algae channelrhodopsin. Proc Natl Acad Sci 102: 17816-17821.

Lin JY, Lin MZ, Steinbach P, Tsien RY. 2009. Characterization of engineered channelrhodopsin variants with improved properties and kinetics. Biophys / 96: 1803-1814.

Lörinczi E, Verhoefen M-K, Wachtveitl J, Woerner AC, Glaubitz C, Engelhard M, Bamberg E, Friedrich T. 2009. Voltage and $\mathrm{pH}$-dependent changes in vectoriality of photocurrents mediated by wild-type and mutant proteorhodopsins upon expression in Xenopus oocytes. I Mol Biol 393: 320-341.

Luecke H, Schobert B, Richter H-T, Cartailler J-P, Lanyi JK. 1999. Structure of bacteriorhodopsin at $1.55 \AA$ resolution. J Mol Biol 291: 899-911.

Man D, Wang W, Sabehi G, Aravind L, Post AF, Massana R, Spudich EN, Spudich JL, Béjà O. 2003. Diversification and spectral tuning in marine proteorhodopsins. EMBO / 22: 1725-1731.

Matsuno-Yagi A, Mukohata Y. 1977. Two possible roles of bacteriorhodopsin; a comparative study of strains of Halobacterium halobium differing in pigmentation. Biochem Biophys Res Commun 78: 237-243.

Michel H, Oesterhelt D. 1976. Light-induced changes of the $\mathrm{pH}$ gradient and the membrane potential in $H$. halobium. FEBS Lett 65: 175-178.

Miesenböck G, Kevrekidis IG. 2005. Optical imaging and control of genetically designated neurons in functioning circuits. Annu Rev Neurosci 28: 533-563.

Nagel G, Ollig D, Fuhrmann M, Kateriya S, Musti AM, Bamberg E, Hegemann P. 2002. Channelrhodopsin-1: A lightgated proton channel in green algae. Science 296: 2395-2398.

Nagel G, Szellas T, Huhn W, Kateriya S, Adeishvili N, Berthold P, Ollig D, Hegemann P, Bamberg E. 2003. Channelrhodopsin-2, a directly light-gated cation-selective membrane channel. Proc Natl Acad Sci 100: 13940-13945.

Nagel G, Brauner M, Liewald JF, Adeishvili N, Bamberg E, Gottschalk A. 2005. Light activation of channelrhodopsin-2 in excitable cells of Caenorhabditis elegans triggers rapid behavioral responses. Curr Biol 15: 2279-2284.

Oesterhelt D, Hess B. 1973. Reversible photolysis of the purple complex in the purple membrane of Halobacterium halobium. Eur J Biochem 37: 316-326.

Oesterhelt D, Stoeckenius W. 1971. Rhodopsin-like protein from the purple membrane of Halobacterium halobium. Nat New Biol 233 $149-152$.

Oesterhelt D, Hegemann P, Tittor J. 1985. The photocycle of the chloride pump halorhodopsin. II: Quantum yields and a kinetic model. EMBO / 4: 2351-2356.

Perálvarez-Marin A, Márquez M, Bourdelande J-L, Querol E, Padrós E. 2004. Thr-90 plays a vital role in the structure and function of bacteriorhodopsin. / Biol Chem 279: 16403-16409.

Racker E, Stoeckenius W. 1974. Reconstitution of purple membrane vesicles catalyzing light-driven proton uptake and adenosine triphosphate formation. J Biol Chem 249: 662-663. 
Radu I, Bamann C, Nack M, Nagel G, Bamberg E, Heberle J. 2009. Conformational changes of channelrhodopsin-2. I Am Chem Soc 131: 7313-7319.

Rickgauer JP, Tank DW. 2009. Two-photon excitation of channelrhodopsin-2 at saturation. Proc Natl Acad Sci 106: 1502515030.

Ritter E, Stehfest K, Berndt A, Hegemann P, Bartl FJ. 2008. Monitoring light-induced structural changes of channelrhodopsin-2 by UV-visible and Fourier transform infrared spectroscopy. I Biol Chem 283: 35033-35041.

Sabehi G, Kirkup BC, Rozenberg M, Stambler N, Polz MF, Béjà O. 2007. Adaptation and spectral tuning in divergent marine proteorhodopsins from the eastern Mediterranean and the Sargasso Seas. ISME J 1: 48-55.

Sakmar TP. 2002. Structure of rhodopsin and the superfamily of sevenhelical receptors: The same and not the same. Curr Opin Cell Biol 14: 189-195.

Scharf B, Engelhard M. 1994. Blue halorhodopsin from Natronobacterium pharaonis: Wavelength regulation by anions. Biochemistry 33: 6387-6393.

Schobert B, Lanyi JK. 1982. Halorhodopsin is a light-driven chloride pump. J Biol Chem 257: 10306-10313.

Schoenenberger P, Gerosa D, Oertner TG. 2009. Temporal control of immediate early gene induction by light. PLoS One 4: e8185. doi: 10.1371 /journal.pone.0008185.

Shichida Y, Yamashita T. 2003. Diversity of visual pigments from the viewpoint of $\mathrm{G}$ protein activation-comparison with other $\mathrm{G}$ protein-coupled receptors. Photochem Photobiol Sci 2: 12371246.

Sineshchekov OA, Jung K-H, Spudich JL. 2002. Two rhodopsins mediate phototaxis to low- and high-intensity light in Chlamydomonas reinhardtii. Proc Natl Acad Sci 99: 8689-8694

Spudich JL. 1998. Variations on a molecular switch: Transport and sensory signalling by archaeal rhodopsins. Mol Microbiol 28: $1051-1058$.

Spudich JL. 2006. The multitalented microbial sensory rhodopsins. Trends Microbiol 14: 480-487.
Spudich JL, Bogomolni RA. 1984. Mechanism of colour discrimination by a bacterial sensory rhodopsin. Nature 312: 509-513.

Stehfest K, Ritter E, Berndt A, Bartl F, Hegemann P. 2010. The branched photocycle of the slow cycling Channel-rhodopsin-2-C128T. J Mol Biol 398: 690-702.

Stoeckenius W, Lozier RH, Niederberger W. 1977. Photoreactions of bacteriorhodopsin. Biophys Struct Mech 3: 65-68.

Suzuki T, Yamasaki K, Fujita S, Oda K, Iseki M, Yoshida K, Watanabe M, Daiyasu H, Toh H, Asamizu E, et al. 2003. Archaeal-type rhodopsins in Chlamydomonas: Model structure and intracellular localization. Biochem Biophys Res Commun 301: 711-717.

Takahashi T, Mochizuki Y, Kamo N, Kobatake Y. 1985. Evidence that the long-lifetime photointermediate of s-rhodopsin is a receptor for negative phototaxis in Halobacterium halobium. Biochem Biophys Res Commun 127: 99-105.

Tsunoda SP, Hegemann P. 2009. Glu 87 of channelrhodopsin-1 causes $\mathrm{pH}$-dependent color tuning and fast photocurrent inactivation. Photochem Photobiol 85: 564-569.

Váró G, Brown LS, Lakatos M, Lanyi JK. 2003. Characterization of the photochemical reaction cycle of proteo-rhodopsin. Biophys / 84: 1202-1207.

Wang W-W, Sineshchekov OA, Spudich EN, Spudich JL. 2003. Spectroscopic and photochemical characterization of a deep ocean proteorhodopsin. J Biol Chem 278: 33985-33991.

Wang H, Sugiyama Y, Hikima T, Sugano E, Tomita H, Takahashi T, Ishizuka T, Yawo H. 2009. Molecular determinants differentiating photocurrent properties of two channelrhodopsins from Chlamydomonas. J Biol Chem 284: 5685-5696.

Yoshitsugu M, Yamada J, Kandori H. 2009. Color-changing mutation in the E-F loop of proteorhodopsin. Biochemistry 48: 4324-4330.

Zhang F, Wang L-P, Brauner M, Liewald JF, Kay K, Watzke N, Wood PG, Bamberg E, Nagel G, Gottschalk A, et al. 2007. Multimodal fast optical interrogation of neural circuitry. Nature 446: 633-639.

Zhang F, Prigge M, Beyrière F, Tsunoda SP, Mattis J, Yizhar O, Hegemann P, Deisseroth K. 2008. Red-shifted optogenetic excitation: A tool for fast neural control derived from Volvox carteri. Nat Neurosci 11: $631-633$ 


\section{Microbial Opsins: A Family of Single-Component Tools for Optical Control of Neural Activity}

Ofer Yizhar, Lief Fenno, Feng Zhang, Peter Hegemann and Karl Diesseroth

Cold Spring Harb Protoc; doi: 10.1101/pdb.top102

\begin{tabular}{rc}
$\begin{array}{r}\text { Email Alerting } \\
\text { Service }\end{array}$ & Receive free email alerts when new articles cite this article - click here. \\
\hline $\begin{array}{c}\text { Subject } \\
\text { Categories }\end{array}$ & $\begin{array}{c}\text { Browse articles on similar topics from Cold Spring Harbor Protocols. } \\
\text { Cell Biology, general (1382 articles) }\end{array}$ \\
& $\begin{array}{c}\text { Cell Imaging (525 articles) } \\
\text { Imaging for Neuroscience (342 articles) } \\
\text { Imaging/Microscopy, general (579 articles) } \\
\text { In Vivo Imaging (334 articles) } \\
\text { In Vivo Imaging, general (168 articles) } \\
\text { Neuroscience, general (357 articles) } \\
\text { Photoactivation (37 articles) }\end{array}$ \\
\hline
\end{tabular}

